\title{
Paternité. \\ Figures du père et lien de filiation
}

\author{
Jean GUYOTAT
}

14 rue Sour Bouvier, Lyon

\section{RESUME}

Etre père c'est s'inscrire et être inscrit dans un réseau de filiation, c'est-à-dire se situer et être situé de façon nouvelle par rapport à ses ascendants et descendants, réels et imaginaires. Cette inscription ne va pas sans une certaine modification de l'identité personnelle dont les modalités sont étudiées à partir de ce que nous enseigne la psychopathologie du lien de filiation. Pour les comprendre des indications sont données sur la structure de ce lien. Les modifications culturelles actuelles de l'Image du père sont aussi abordées.

Mots-clés : Institution - paternité - identité - narcissisme - père biologique - filiation - psychose.

On assiste depuis plusieurs années à une modification dans notre culture de la figure du père. De nombreux colloques ont été consacrés à l'étude de cette transformation. Par exemple dans une réunion tenue à Paris en 1988 Evelyne SULLEROT se posait la question que beaucoup d'autres avaient évoquée : Allons nous vers une société sans père? D'où de nombreux ouvrages consacrés à la question dont l'un de référence" Le Père" dans le cadre de l'Espace Analytique [5]. Cette question concerne au plus haut point aussi la psychanalyse et on ne peut aborder la question de la paternité sans prendre en compte les variations de l'image sociale car en matière de paternité les modèles ont une particulière importance.
Parmi les raisons invoquées à l'origine de cette transformation sociale, je n'en retiendrai que deux.

Celle des apports de la biologie de la reproduction dans la représentation collective de la problématique paternelle. Il devient classique de dire que l'on est passé, grâce à ces nouvelles techniques, du schéma mater certissima, pater incertus, presque à l'inverse. Le père est devenu certissimus grâce à la méthode des empreintes génétiques. Quant à la mère, elle n'est plus obligatoirement celle qui accouche de l'enfant, la gestratrix, car elle peut porter en son sein un fœtus né d'un ovule étranger à elle, d'une génitrix dans la technique du don d'ovule. Quant à l'insémination artificielle par donneur anonyme, qui reste contestée par certains, elle propose de façon officielle dans un cadre médicalisé la dissociation entre le géniteur, le donneur de sperme anonyme et le père au sens légal, institué du terme. Ces transformations ont un écho important dans l'opinion publique et chez les techniciens sont l'objet de réflexions bioéthiques, elles mêmes génératrices de propositions législatives.

L'autre raison de cette modification de l'image du père agit plutôt en sens inverse. C'est celle qui résulte de la situation d'exil, tant pour les exilés que pour les autochtones parmi lesquels ils vivent, qui introduit une coupure d'avec les représentations traditionnelles de la figure du père. On peut prendre comme exemple le cas de l'immi- 
gration maghrébine et la rencontre qu'elle opère entre civilisation occidentale et l'Islam. F. BENSLAMA [2], dans un ouvrage récent" Une fiction troublante, de l'origine en partage", essaie d'analyser la nature blasphématoire pour les intégristes musulmans de l'ouvrage de Salman RusHdIE" Les versets sataniques". Il fait allusion aux peuples qui ne se laisseront pas" déligitimer généalogiquement" et montre que le délit a un corps," le corps du texte mythique du père" qu'attaquerait le romancier. Bien que n'acceptant en aucun cas la sentence, c'està-dire la mise à mort du romancier, il montre combien il s'agit d'un conflit entre l'universel (par exemple celui de la Déclaration Universelle des Droits de l'Homme et d'une façon générale celle de la civilisation occidentale) et l'originaire, lequel est en étroite relation avec le mythe paternel.

Culturellement le conflit se situe entre deux façons de traiter ou de maltraiter la figure du père, étant entendu que l'une fonctionne comme un traumatisme psychique pour l'autre. Le croyant est quelque part psychiquement traumatique pour l'incroyant et inversement. La situation d'exil serait traumatique à ce point précis, celui du mythe du père.

Il est donc intéressant de voir comment fonctionne cette figure du père par rapport au lien de filiation.

A partir de recherches concernant tant la psychopathologie de la filiation que celle de la puerpéralité et de la paternité, j'ai proposé la définition suivante du lien de filiation : ce par quoi un individu se situe et est situé par rapport à ses ascendants et ses descendants réels et imaginaires.

Cette définition marque :

- le caractère ascendant et descendant du lien de filiation. En français le mot filiation renvoie peut être plus à l'ascendance alors qu'en anglais ce sera l'inverse, ce qui correspondrait alors plus à ce que nous appelons nous en français descendance.
- le caractère individuel et groupal du lien. On est situé par le groupe comme étant le père, le fils de mais on se situe soit même comme tel, étant entendu que cette façon de se situer personnellement est très liée à la construction de l'identité. Par exemple le rejet du père, l'affirmation de soi comme son propre père, comme étant auto-généré, fréquent à l'adolescence, est souvent le signe d'une difficulté identitaire que l'on résoudra éventuellement par l'immersion dans un groupe déviant. Et le fait de devenir pour la première fois père peut relancer ces difficultés d'identité.

Le caractère réel et imaginaire du lien est lui même important à considérer. Nous connaissons bien en psychiatrie ces constructions délirantes que font certains patients à propos de leurs ascendants : substitution au père" réel" (c'est-à-dire celui qui est institué juridiquement par : le nom (nomen), la réputation (fama), la possession d'état (tractatus) d'un ancêtre paternel imaginaire prestigieux, souvent mort de mort violente (Louis XVI, le Tsar Nicolas... rarement Hitler), sans descendance. Il est moins classique de parler des substitutions d'enfant à la naissance que commencent à connaître les biologistes qui s'occupent d'empreintes génétiques [3]. On peut remarquer que c'est surtout le père qui est l'objet de cette substitution du côté ascendant, alors que du côté descendant c'est surtout la mère qui proclame cette substitution. La figure du père est de préférence projetée sur l'ascendance, l'originaire, celle de la mère sur la descendance. Il y a un lien entre l'originaire et la figure du père.

On peut aller plus loin et tenter d'analyser la structure du lien de filiation.

J'ai déjà fait allusion au lien institué en citant au passage quelques formules juridiques classiques. Tout individu est inscrit par le groupe auquel il appartient, qu'il le veuille ou non, dans un ordre symbolique, langagier ou non langagier. Les psychanalystes lacaniens ont beaucoup développé cette problématique avec la formule" le nom 
du père". La transmission du nom qui se trouve dans nos sociétés être celui du père aurait une importance considérable dans la structuration de l'individu. On connaît les troubles entraînés par des changements intempestifs de patronyme dans la mesure où ils sont vécus par l'enfant comme une attaque de la figure du père, c'est à dire comme une attaque par la mère de sa propre croyance au père. Car le père, c'est un mythe auquel on croit. C'est la parole de la mère qui dit :" cet enfant est de toi", qu'elle est la seule à pouvoir dire en principe. Mais au delà de l'affirmation de la mère il y a la transmission généalogique du nom :" qu'est-ce qu'une généalogie? C'est le sillage onomastique de la vie mue au travers des morts" (St Zagdanski).

Cependant l'ordre symbolique n'est pas que langagier. Il se manifeste aussi à travers l'organisation institutionnelle, institution que l'on peut définir comme" un dispositif organisé visant au fonctionnement ou à la reproduction d'une société donnée, résultat d'une volonté originelle (acte d'instituer et d'une adhésion à sa légitimité supposée)" [1]. Concernant le lien de filiation, il y a par exemple les problèmes de la transmission des biens, les règles d'appartenance de l'enfant à l'un ou l'autre de ses parents qui prennent une importance considérable en cas de contestation. On connaît en cas de divorce le problème des pères à qui une décision judiciaire à enlevé la garde de l'enfant. Dans ces cas à la douleur de la séparation d'avec l'enfant s'ajoute l'attaque de la figure du père qui est en elle même déstructurante.

Cependant à un autre niveau on peut constater, et c'est le cas notamment à propos de la paternité biologique, que l'affaiblissement dans notre culture de la figure du père s'accompagne d'une inflation de la réflexion éthique et d'un développement en ce qui concerne la pratique des comités de bioéthiques. Il y a donc un lien indiscutable entre l'organisation instituée, l'ordre symbolique et la figure du père. Plus celle-ci s'affaiblit, plus les institutions tendent à accroître leur rôle.

Le temps n'est donc plus où juridiquement le père c'était le mari de la mère qui définissait la position symbolique du père par rapport à la mère, vis-à-vis de l'enfant avec laquelle elle a une relation (métonymique) de proximité de corps à corps. Cependant la figure du père reste traditionnellement rattachée à l'ordre symbolique, à l'institution, et on ne saurait imaginer une société qui soit complètement coupée de ses traditions et on peut même dire que l'éthique c'est aussi le maintien de la tradition dans l'actuel et on voit bien que c'est ce qui se passe dans des discours comme ceux qui ont eu lieu à propos de la bioéthique, par exemple dans le rapport de N. LENOIR" Aux portes de la vie: paroles d'éthiques" [3]. Le mythe paternel y reste en tant que référence originaire toujours actif dans les prises de position.

J'ai fait allusion plus haut à propos de la mère au lien de corps à corps. On peut en effet décrire un lien de filiation de corps à corps qui est, dans ma perspective, celui qui inspire la logique du lien biologique. On peut en effet dire que le lien du corps de l'enfant au corps de la mère procède de la même logique que celle des rapports entre les morceaux et les produits du corps. Toute la biologie s'édifie sur ce type de rapport dans la mesure ou c'est seulement ce type de lien qui est considéré ou privilégié ; on peut alors parler selon l'expression de $P$. LEGENDRE d'une filliation bouchère dont l'expérience nazie nous a donné un exemple dramatique. On se rappelle en effet que le nazisme a pu être décrit comme la biologie appliquée au politique. Il semble bien que des réticences ou des inquiétudes vis-à-vis des manipulations génétiques ou des procréations médicalement assistées relèvent de la persistance de cette stigmatisation du lien biologique comme d'essence raciste, ce qui aurait donc une origine historique. Mais on peut remarquer aussi que le lien de filiation porte en lui les prémices de son éclate- 
ment aux dépens de l'un ou l'autre de ses constituants, tant il s'agit d'une formation hétérogène. Quoi de plus hétérogène en effet que des logiques qui sont celles de la biologie et celles du symbolique. Quoi de plus hétéroclite que la conjonction sur un même sujet de la réalité de son corps mortel et de celle de son nom, celui de son père. On peut donc s'attendre concernant cette structure du lien de filiation à de continuels renversements.

Pour les comprendre de plus près il est nécessaire d'introduire une autre logique, celle de la filiation narcissique.

Beaucoup d'arguments cliniques et psychologiques sont en faveur de cette dimension narcissique.

En psychiatrie il y a des figures cliniques qui peuvent être considérées comme une inflation de l'image de soi qui caractérise le narcissisme dont on rappelle la définition psychanalytique très éclairante :" amour porté à l'image de soi" (LAPLANChe et Pontalis). Le délire de filiation en est l'exemple caractéristique. Du côté de la descendance il apparaît bien que, dans la psychose puerpérale typique, la patiente remplace l'enfant réel qui vient de naître par un enfant imaginaire qui est le double d'elle même, d'où parfois la mise à mort de l'enfant réel et le suicide de la mère pour maintenir cette construction imaginaire à la fois exaltante et mortifêre. On peut donc dire que le lien de filiation narcissique est structuré autour du fantasme de la reproduction du même, comme fantasme d'immortalité tout mortifere qu'il soit. FREUD a bien montré, dans le Roman familial des névrosés, comment tout enfant vers 5 ans passe par une phase où il remplace ses parents réels par des parents imaginaires plus satisfaisants pour l'image qu'ils lui renvoient. La force de ce lien imaginaire on la retrouve dans beaucoup de mythes, ceux du double, ceux de la reproduction par scissiparité, clonique, qui existe dans la nature mais, en ce qui concerne l'espèce humaine, essentiellement dans les mythes.
Dans les psychoses de la paternité par identification à la mère, le père peut vivre la naissance de son enfant sur le même mode.

Le lien narcissique imaginaire investit la lignée aux dépens de la filiation née de l'alliance d'une homme et d'une femme, d'un père et d'une mère (qui est celui de la définition juridique de la filiation). En anthropologie on décrit des organisations sociales appelées groupe de filiation dont font partie tous les individus issus d'un ancêtre commun. Dans le système patrilinéaire par exemple, la mère ne fait pas partie de la même lignée que son fils alors que c'est le cas du père. C'est la dimension verticale du lien à partir d'une origine commune qui est investie.

Quelle est l'image du père qui apparaît alors?

Il semble, et j'ai pu avec d'autres le constater dans les délires de filiation, que chaque fois qu'il y a un manque de la filiation instituée (père inconnu, enfant de l'assistance publique, adoption dans la mesure ou il y a écart entre le lien de sang biologique et le lien institué, l'insémination artificielle par donneur etc...) le lien narcissique soit exalté. C'est donc une image du père exalté qui se constituera à la recherche d'un père idéal qui pourrait être :

- soit le père de la horde primitive, du mythe Freudien ou le leader totalitaire d'un groupe ou d'une nation.

- soit une figure qui procède de la même aspiration mais qui s'organise autour d'une pôle religieux, lequel se construit à partir d'un texte révélé originaire (Bible, Coran...) et lie étroitement la séquence des engendrements et le texte originaire de la révélation. Le système religieux réintroduit le lien institué par le texte (biblique...) alors que le père totalitaire est celui qui édicte lui même ses propres textes au mépris de la tradition. Il est ce père qui s'engendre lui même.

Qu'est-ce que le mot biologique modifie dans la figure du père? On ne reviendra pas sur biologie et politique. On utilise de 
plus en plus dans les écrits notamment journalistiques le terme de père biologique. N'y a t-il pas sur cette expression projection d'une sorte de père idéal surgi des origines qui supporterait paradoxalement tout ce qui ne ressort pas de la problématique paternelle instituée et qui se nourrit de ses manques. Peut-on éviter de retomber par extension dans cette dichotomie corps esprit lorsque l'on aborde la relation du biologique à l'institué ?

A titre d'exemple je partirai de la représentation du sperme. Il est intéressant de remarquer avec Giulia Sissa [5] dans un article" Le paternel comme principe" ce qui s'est discuté autour de la notion de génération spontanée. L'auteur fait remarquer que l'on trouve aussi bien dans la génétique sauvage des trobiandais que dans Aristote le principe" soustraire le statut paternel véhiculé par le sperme aux contraintes de la matérialité". Le sperme est un souffle qui donne vie à la matrice, celle qui git dans l'utérus de la femme et l'auteur remarque que, jusqu'aux fameuses expériences de Pouchet et PAsteur, a persisté sous une forme religieuse la concordance du sperme de l'homme avec quelque chose d'immatériel.

Le fantasme est probablement tout-à-fait à l'action dans la problématique de l'insémination post-mortem. Sur le sperme congelé du mari mort, le groupe projette quelque chose qui est de l'ordre de l'immatériel qui peut maintenir celle qui fait la demande dans un deuil infini. Cette croyance émouvante n'en est pas moins problématique pour le juriste qui déclare" toute entreprise d'immortalité est contraire à l'ordre public". Chassez l'institué, il revient au galop

Mais il est intéressant de voir quelles peuvent être les conséquences sur la Recherche d'un tel fantasme. Dans un interview récent publié par le Journal Le Monde (6/7/94), de A. VAN et P. DEVRoey à propos de la microinjection du spermatozoïde comme mode de traitement de la stérilité masculine, il est bien montré que l'un des critères classiques retenus dans l'analyse de la qualité du spermogramme, la mobilité du spermatozoïde, était purement imaginaire. Un spermatozoïde peu mobile d'après ces chercheurs peut fort bien transporter un contenu génétique tout-à-fait actif. On pourrait dire que lon a projeté longtemps sur le spermatozoïde repéré dans le spermogramme, l'image primitive du souffle qui donne vigueur et mobilité.

En pathologie mentale il y a bien souvent corrélations entre manque dans la filiation instituée, image du père et refus de sa propre paternité. C'est le cas chez ce patient portugais où l'image du père véhiculé comme il est fréquent par le frère aîné, engendre une contestation chez lui de sa propre paternité. Chez ce patient le fait que son frère aîné soit diabétique et stérile entraîne un délire momentané de refus de la paternité de ses propres enfants. Il ne reconnaît plus le visage de ses propres enfants.

Dans une autre observation clinique, le père du sujet était hémiplégique depuis de nombreuses années dès l'enfance de son fils. Or ce fils, lorsqu'il devient père d'un garçon, fait à son tour une dépression avec tentative de suicide. Il ne s'agit pas de retenir cette seule étiologie mais d'essayer de s'en servir pour essayer de comprendre avec le malade le sens de son geste.

Dans ces deux brefs exemples on voit que quelque chose s'est passé par défaut de symbolisation. L'anomalie biologique de l'ascendant ou de son représentant passe en quelque sorte directement dans l'appréciation du réel de la descendance.

La figure du père parait donc plus que la mère inscrite dans ce réseau institué et toute singularité de ce réseau va mettre en route la dimension imaginaire narcissique du lien, d'où le délire ou une dépression ou peut être un retentissement psychosomatique qui demanderait à être mieux évalué.

Lorsque les repères institués bougent et c'est le cas lors d'une naturalisation, d'un 
changement culturel de l'exil, la figure du père est attaquée ou $\mathrm{du}$ moins mise en cause. Beaucoup d'épisodes délirants plus souvent dépressifs avec violence sont en rapport avec la perte pour le père de ses rapports institués lorsque le fils s'inscrit plus précisément dans la culture du pays d'accueil ou refuse de s'y inscrire. Par exemple dans le mariage, autre institution, la substitution du rôle du père à celui du frère aîné, comme je l'ai dit plus haut, est quelquefois aussi pathogène, par exemple lorsque le frère aîné ne peut assumer le rôle paternel lorsque son propre père tombe malade ou est en chômage.

D’après ma façon de voir les choses, je parlerai volontiers dans ce cas de blessure institutionnelle du lien de filiation, cette blessure pouvant provenir de la part prise à un moment donné chez cet autrui par le biologique, le corps, dans l'histoire d'une famille comme on l'a vu dans l'histoire du père hémiplégique.

La paternité est définie dans les dictionnaires comme état, qualité de père. Il existe donc une paternité biologique, quant à la paternité psychologique elle se constitue progressivement dans les relations du sujet avec ses propres enfants et procède souvent de la relation de ce sujet avec son propre père que la psychanalyse a beaucoup étudiée à travers la problématique œdipienne, ce qui est bien connu.

Cette paternité s'inscrit aussi comme on l'a vu dans la chaîne des générations, c'est-àdire à travers les vicissitudes des liens de filiation.

\section{REFERENCES}

1. Augustins G. : Institution. In Dictionnaire de l'Ethnologie et de l'Anthropologie. Pierre Boule, Michel Izard, PUF, 1991.

2. BENSLAMA F. : Une fiction troublante de l'origine en partage. Edition de l'Auge, 1994, : 1 vo. 93pp.

3. DupRET D. : Empreintes génétiques et filiation. Rapport N. Lenoir. Aux frontières de la vie : paroles d'éthique. La Documentation Française, 1991.

4. Guyotat J. : Etudes cliniques d'Anthropologie Psychiatrique. Masson Editions, 1991.

5. Sissa G. : Arche Kimoussa ou le paternel comme principe. In le Père. Métaphore paternelle et fonction du père : l'interdit, la filiation, la Transmission. L'Espace Psychanalytique. Ed. Denoel, 1989 : 147-163.

6. ZAGDANSKI S. : L'impureté de Dieu. La lettre et le péché dans la pensée juive. Edition Felin, 1991.

\section{ABSTRACT \\ Paternity. Images of the father and affiliation ties}

Jean GuyotaT

To be father is to fit into and enter a network of affiliation, that is to occupy and take up a new position with respect to his ancestries and progenies real or imaginary. The enrolment goes naturally with a certain modification of the personal identity and the teachings of the psychopathology of the affiliation ties learns us its modalities. Indications are given on the structure of this ties. The actual cultural modifications of the Image of the father are also tackled.

Key words : Institution, paternity, identity, narcissism, biological father, affiliation. 